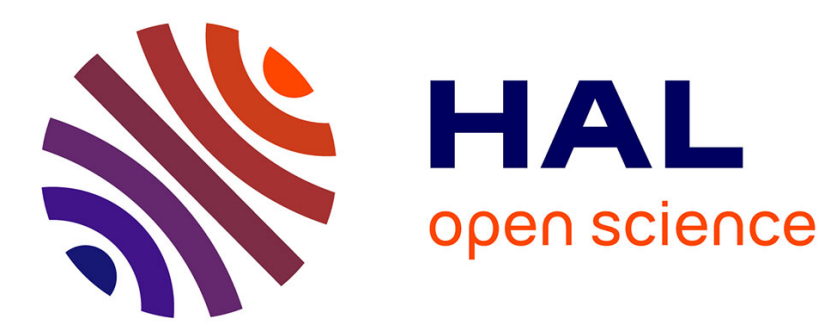

\title{
On the higher order mode coupler design for damped accelerating structures
}

J. Gao

\section{To cite this version:}

J. Gao. On the higher order mode coupler design for damped accelerating structures. 16th IEEE Particle Accelerator Conference (PAC 95) and International Conference on High-energy Accelerators (IUPAP), May 1995, Dallas, United States. pp.1717-1719, 10.1109/PAC.1995.505338 . in2p3-00022709

\section{HAL Id: in2p3-00022709 https://hal.in2p3.fr/in2p3-00022709}

Submitted on 5 May 2008

HAL is a multi-disciplinary open access archive for the deposit and dissemination of scientific research documents, whether they are published or not. The documents may come from teaching and research institutions in France or abroad, or from public or private research centers.
L'archive ouverte pluridisciplinaire HAL, est destinée au dépôt et à la diffusion de documents scientifiques de niveau recherche, publiés ou non, émanant des établissements d'enseignement et de recherche français ou étrangers, des laboratoires publics ou privés. 


\title{
On the Higher Order Mode Coupler Design for Damped Accelerating Structures
}

\author{
Jie Gao

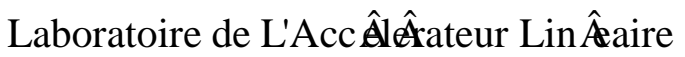 \\ IN2P3-CNRS et Universitêtde Paris-Sud \\ 91405 Orsay cedex, France
}

\begin{abstract}
In this paper we will discuss mainly damped structures and class them into three types. The procedures to make a higher order mode (HOM) coupler design have been given, and some useful analytical formulae, which can be used to determine the coupling apertures' dimensions and the equivalent loaded quality factors, have been derived. It is shown that few couplers to dampe a detuned structure is not at all ef®cient.
\end{abstract}

\section{Introduction}

For the linear colliders working in the multibunch mode the long range wake®elds have to be properly controlled by using detuned, damped or damped detuned structures depending on the concrete machine design parameters. In this paper we try to class damped structures into three types and to make a detail discussion about how to determine the coupling aperture dimension of a HOM coupler and how to estimate its effect on the damped HOM. In section 2, the ®rst type damped structure is discussed, where each cavity is damped by HOM couplers. In section 3, the second type damped structure is deßned as a section of constant impedance structure is damped by a HOM coupler. The essential difference between the Brst and the second type damped structures is demonstrated. To estimate the effect of this HOM coupler an equivalent quality factor has been introduced and formularized. Finally, in section 4, damped detuned structures are discussed and it is shown that few couplers to dampe a detuned structure is not at all ef®cient.

\section{The First Type of Damped Structure}

We consider a single pill-box resonant rf cavity resonating at a resonant mode, for example the $\mathrm{TM}_{110}$ mode. The quality factor corresponding to this mode is $Q_{110}$ If this cavity is loaded with some waveguides, the loaded quality factor $Q_{L, 110}$ is deßned as

$$
Q_{L, 110}=\frac{Q_{0,110}}{1+\beta_{N, 110}}
$$

where $\beta_{N, 110}$ is called the coupling coef $®$ cient between waveguides and the resonant cavity corresponding to the $\mathrm{TM}_{110}$ mode. Where the subscript $N$ denotes the number of the waveguides. If the cavity is loaded with four waveguides distributed uniformly around the cavity's cylindrical surf ace, from the general expression derived in refs. [1] and [2] one ${ }^{\circledR}$ nds that $\beta_{4,110}$ is $\mathbf{T M}_{110}$ mode polarization independent and can be expressed analytically

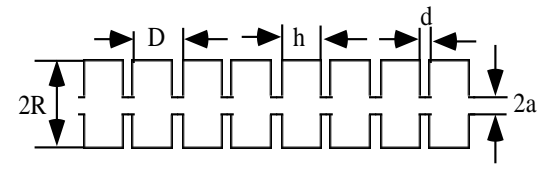

Figure. 1. Constant impedance accelerating structure

as

$$
\beta_{4,110}(l)=\frac{\pi Z_{0} k k_{10} l^{6} e^{-2 \alpha_{c} t} J_{1}^{\prime}\left(u_{11}\right)^{2}}{144\left(\ln \left(\frac{4 l}{w}\right)-1\right)^{2} A B R R_{s, 110}(R+h) J_{2}^{2}\left(u_{11}\right)}
$$

where $k=2 \pi / \lambda_{110}, k_{10}=k\left(1-\left(\lambda_{110} / 2 A\right)^{2}\right)^{1 / 2}, \alpha_{c}=$ $\left(2 \pi / \lambda_{110}\right)\left(\left(\lambda_{110} / 2 l\right)^{2}-1\right)^{1 / 2}, R_{s, 110}=\left(\pi c \mu_{0} / \sigma \lambda_{110}\right)^{1 / 2}, \mu_{0}$ is the magnetic permeability and $\sigma$ is the electric conductivity, $A$ and $B$ are the width and the height of HOM waveguides, $l$ and $w$ are the width and the height of the four rectangular coupling slots with $l$ parallel to the magnetic ${ }^{\circledR} e l d$, and $t$ is the wall thickness between cavity inner surface and waveguide. Now, if many of this kind of waveguide loaded cavities are coupled together, one obtains the ®rst kind of damped accelerating structureunder the condition that the fundamental mode is not damped. For the $\mathrm{TM}_{11}$ mode passband one can say that the loaded $Q$ is almost $Q_{L, 110}$. If $K_{h} Q_{L, 110}<2$ there will be no coupling between cavities for the $\mathrm{TM}_{11}$ mode [3], where $K_{h}$ is the coupling coefßcient for the $\mathrm{TM}_{11}$ mode passband.

\section{The Second Type of Damped Structure}

Now we consider the second kind of damped structure which is essentially different from the ${ }^{\circledR}$ rst. Given a constant impedance disk-loaded structure of length $L$ as shown in Fig. 1 , one can calculate the passband of the $\mathrm{TM}_{11}$ mode (which is the most dangerous mode for multibunch operation). We de®ne $\theta_{1, s}$ as the phase shift of the $\mathrm{TM}_{11}$ mode passband at which the phase velocity equals to the velocity of light. Assuming now a charged particle is injected into the structure off axis at a velocity close to that of light, one knows that this particle will loss its energy to the $\mathrm{TM}_{11}$ mode mainly at synchronous phase shift $\theta_{1, s}$ and generates behind it so-called wake®eld $W_{\perp, 11}(s)$, where $s$ is the distance between this exciting particle and a following test particle (of course it will generate wake®elds oscillating at other 


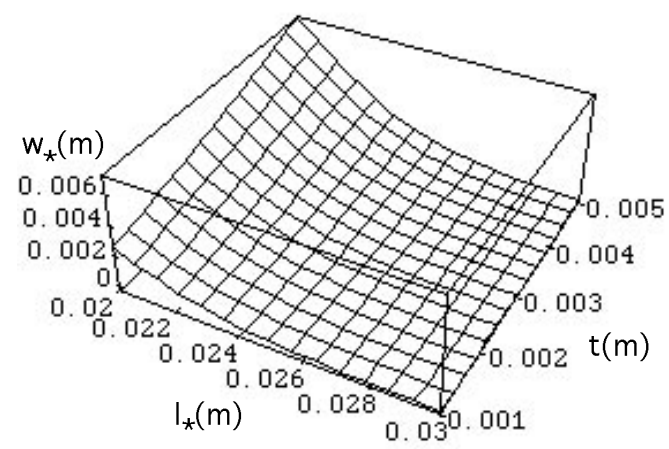

Figure. 2. $\beta_{4,11}=1$ surface with $v_{g}\left(\theta_{1, s}\right) / c=0.001$

frequencies also). If $0<\theta_{1, s}<\pi$ the energy of the wake®eld $W_{\perp, 11}$, which is distributed uniformly along the structure length $L$ just after the passage of the exciting electron, will tend to move upstream with the group velocity of $v_{g}\left(\theta_{1, s}\right)$. Assuming that four distributed waveguides, for example, are connected to the ${ }^{\circledR}$ rst cavity at the beginning of the structure, in order to absorb the energy of $W_{\perp, 11}$ from the structure via the HOM waveguides without any re ${ }^{-}$ection, one has to match this HOM coupler to the $\mathrm{TM}_{11}$ travelling wave mode working at $\theta_{1, s}$ by carefully choosing the slot dimensions on the coupler wall (just like matching the fundamental mode input and output couplers of a constant impedance travelling wave accelerating structure). The coupling slots' width $l_{*}$ and height $w_{*}$ have to satisfy the following relation [2][4]:

$$
\begin{gathered}
\beta_{4,11}\left(l_{*}\right)=\frac{\pi Z_{0} k k_{10} l_{*}^{6} \exp \left(-2 \alpha_{c}^{*} t\right) J_{1}^{\prime}\left(u_{11}\right)^{2}}{144\left(\ln \left(\frac{4 l_{*}}{w_{*}}\right)-1\right)^{2} A B R R_{s, 110}(R+h) J_{2}^{2}\left(u_{11}\right)} \times \\
\frac{1}{\left(1+\frac{Z_{0} R}{2 R_{s, 110}(R+h)}\left(\frac{v_{s}\left(\theta_{1, s}\right)}{c}\right)\right)}=1
\end{gathered}
$$

where $\alpha_{c}^{*}=\left(2 \pi / \lambda_{110}\right)\left(\left(\lambda_{110} / 2 l_{*}\right)^{2}-1\right)^{1 / 2}$ and $c$ is the velocity of light. Comparing eq. 2 with eq. 3 , one ${ }^{\circledR}$ nds out the fundamental difference between the two kinds of damped structure, that is, the coupling slots of the HOM coupler of the second type damped structure should not be too large or too small but satisfy matching condition shown in eq. 3 , however, for the ${ }^{\circledR}$ rst type damped structure this limitation doesn't exist. Here we will use eq. 3 to ${ }^{\circledR}$ nd the dimensions of HOM coupling apertures of a $3 \mathrm{GHz}$ accelerating structure with $a=1.5 \mathrm{~cm}, D=3.33 \mathrm{~cm}$, $R=4.1 \mathrm{~cm}, h=2.83 \mathrm{~cm}, A=4.4 \mathrm{~cm}$ and $B=2 . \mathrm{cm}$. Fig. 2 shows the relation between $w_{*}$ and $l_{*}$ for different wall thickness at $v_{g}\left(\theta_{1, s}\right) / c=0.001$.

Once the HOM coupler is matched one knows the de ecting force of the transverse wake ${ }^{\circledR}$ eld $W_{\perp, 11}$ on the test particle of charge $q$ which is behind the exciting particle by a distance of $s$ :

$$
F_{\perp, 11}=q W_{\perp, 11} e^{-\frac{\omega\left(\theta_{1, s}\right)}{2 Q_{0,11}\left(\frac{s}{c}\right)}} D(s)
$$

where

$$
D(s)=L-v_{g}\left(\theta_{1, s}\right) \frac{s}{c},\left(s \leq c L / V_{g}\left(\theta_{1, s}\right)\right)
$$

$$
D(s)=0,\left(s>c L / v_{g}\left(\theta_{1, s}\right)\right)
$$

where $\omega\left(\theta_{1, s}\right)$ is the angular frequency of the travelling $\mathbf{T M}_{11}$ mode at the phase shift of $\theta_{1, s}, Q_{0,11}$ is the quality factor of the structure working at the angular frequency $\omega\left(\theta_{1, s}\right)$. It is obvious that when $v_{g}\left(\theta_{1, s}\right)=0$ there is no damping effect (only the coupler cavity is damped). For those who are used to think in terms of loaded quality factor to judge the damping effect of this HOM coupler, we will use an exponential function $L e^{-\frac{\omega\left(\theta_{1, s}\right)}{2 Q_{e}, 11}\left(\frac{s}{c}\right)}$ to simulate function $D(s)$ in eq. 5 by choosing $Q_{e, 11}$ as

$$
Q_{e, 11}=\frac{\omega\left(\theta_{1, s}\right) L\left(1-e^{-1}\right)}{2 v_{g}\left(\theta_{1, s}\right)}
$$

in the way that both functions drop to the the same value $L e^{-1}$ at the same $s$. Eq. 4 can be expressed as

$$
F_{\perp, 11}=q W_{\perp, 11} L e^{-\frac{\omega\left(\theta_{1, s}\right)}{2 Q_{L, 11}}\left(\frac{s}{c}\right)}
$$

where $Q_{L, 11}$ is called the equivalent loaded quality factor which is

$$
\begin{gathered}
Q_{L, 11}=\frac{Q_{0,11}}{1+\frac{Q_{0,11}}{Q_{e, 11}}} \\
=\frac{Q_{0,11}}{1+\beta_{e q}}
\end{gathered}
$$

where $\beta_{\text {eq }}$ is called equivalent coupling coef $\AA$ cient which has nothing to do with the coupling coef®cients expressed in eq. 2 and eq. 3. If $Q_{0,11} \gg Q_{e, 11}, Q_{L, 11} \approx Q_{e, 11}$. From eq. 6 it is very important to note that there are two ways to decrease $Q_{L, 11}$, either to reduce $L$ or to increase $v_{g}\left(\theta_{1, s}\right)$. The total number $N_{c}$ of the HOM couplers which should be used to reach a desired $Q_{L, 11}^{*}$ for a constant impedance structure of length $L$ can be calculated from the following expression:

$$
N_{c}=\frac{L \omega\left(\theta_{1, s}\right)\left(1-e^{-1}\right)\left(Q_{0,11}-Q_{L, 11}^{*}\right)}{2 c Q_{0,11} Q_{L, 11}^{*}\left(\frac{v_{q}\left(\theta_{1, s}\right)}{c}\right)}
$$

The second kind damped structure is used, for example, in TESLA linear collider project.

\section{The Third Type of Damped Structure}

The third type of damped structure is to damp a detuned structure by few HOM waveguides. We will @ist discuss it without adding HOM coupler and assuming that cavities' dimensions are different from each other adiabatically. The $\mathrm{TM}_{11}$ modes dispersion curves of this detuned structure are shown in Fig. 3. When a particle traverse the structure off axis it will deposit part of its energy on $\mathrm{TM}_{11}$ mode oscillating at the frequencies mainly from $f_{1}$ to $f_{n}$ as shown in Fig. 3, where the subscript $n$ is the total number of different cavities in this detuned structure of length $L$. The de ecting force felt by a test particle can be expressed as

$$
F_{\perp, 11}=\sum_{i=1}^{n} 2 q K_{\perp, 11} \frac{L}{n} \sin \left(\omega\left(\theta_{1, s, i}\right) \frac{s}{c}\right) \exp \left(-\frac{\omega\left(\theta_{1, s, i}\right)}{2 Q_{0,11, i}}\left(\frac{s}{c}\right)\right)
$$

with

$$
K_{\perp, 11}=\left(\frac{k_{1, i} c}{a_{i}^{2} \omega\left(\theta_{1, s, i}\right)}\right)
$$




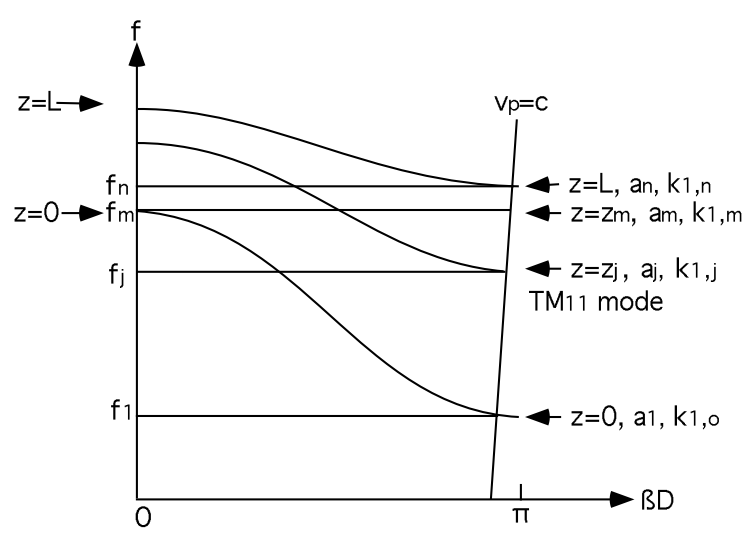

Figure. 3. Dispersion curve of a detuned structure

where $k_{1, i}$ is the $\mathrm{TM}_{11}$ mode loss factor corresponding to the ith cavity, and it can be calculated analytically [2]

$$
\begin{gathered}
k_{1, i}=\frac{a_{i}^{2} u_{11}^{2} D \eta_{\theta_{1, s, i}}^{2}}{4 \pi \epsilon_{0} h R_{i}^{4} J_{2}^{2}\left(u_{11}\right)}\left(\frac{2 R_{i}}{a_{i} u_{11}} J_{1}\left(\frac{u_{11}}{R_{i}} a_{i}\right)\right)^{2} \\
\eta_{\theta_{1, s, i}}=\frac{2}{\theta_{1, s, i}} \sin \left(\frac{\theta_{1, s, i} h}{2 D}\right)
\end{gathered}
$$

If we consider an uniformly detuned structure with its iris radius adiabatically decreasing from $a_{1}$ at the beginning to $a_{n}$ at the end, the deposited energies can move inside the structure instead of oscillating locally. The motions of the $\mathrm{TM}_{11}$ modes' energies inside the structure can be classi®ed into three different ways. For the modes deposited at the beginning of the structure they will oscillate locally since they could not propagate downstream. For the modes generated at the end of the structure (from $f_{m}$ to $\left.f_{n}, v_{g}\left(\theta_{1, s, i}\right)<0, i=m, \cdots, n\right)$, however, the energies will propagate upstream and be trapped $\AA$ nally somewhere in the downstream cavities where the group velocities corresponding to these frequencies are equal to zero. Finally, the energies of the modes between $f_{1}$ and $f_{m}$ will propagate upstream until they are re ${ }^{-}$ected by the ${ }^{\circledR}$ rst cavity. The re ${ }^{-}$ected energies will move downstream and ${ }^{\circledR}$ nally be trapped in the cavities where the group velocities are zero.

To damp the $\mathrm{TM}_{11}$ modes in a detuned structure via a few HOM couplers is the main idea of the so-called damped detuned structure currently adopted in the S-Band linear collider main linac design [5]. To demonstrate the behaviour of a HOM coupler let's assume that at the beginning of a detuned structure a HOM coupler (four waveguides) is installed and is matched to the ith $\mathrm{TM}_{11}$ mode. The equivalent loaded quality factor corresponding to the $i t h$ mode can be expressed as

$$
Q_{L, 11, i}=\frac{Q_{0,11, i}}{1+\frac{Q_{0,11, i} 2<v_{g}\left(\theta_{1, s, i}\right)>}{\omega\left(\theta_{1, s, i}\right) z_{i}\left(1-e^{-1}\right)}}
$$

where $<v_{g}\left(\theta_{1, s, i}\right)>$ is the average group velocity of the $i t h$ mode travelling from $z=z_{i}$ to $z \approx 0$ where the HOM coupler is located. The damping effects of this HOM coupler on the other modes, however, are less ef®cient and different from one to another due to mismatching. The re ${ }^{-} e c t e d$ HOM energies by this mismatched HOM coupler will be trapped somewhere in the structure. It seems that the ${ }^{\circledR}$ rst type damping scheme is the most suitable choice for a damped detuned structure (SLAC damped detuned structure is a good example [6]).

\section{Acknowledgements}

He appreciates the discussions with J. Le Duff and H. Braun.

\section{References}

[1] J. Gao, ${ }^{\circ}$ Analytical formula for the coupling coef®cient $\beta$ of a cavity-waveguide coupling system ${ }^{\circ}$, Nucl. Instr. and Meth., A309 (1991) p. 5.

[2] J. Gao, ${ }^{\circ}$ Analytical approach and scaling laws in the design of disk-loaded travelling wave accelerating structures ${ }^{\circ}, \mathrm{Par}^{-}$ ticle Accelerators, Vol. 43(4) (1994) pp. 235-257.

[3] J. Gao, ${ }^{\circ}$ The criterion for the coupling states between cavities with losses ${ }^{\circ}$, Nucl. Instr. and Meth., A352 (1995) pp. 661-662.

[4] J. Gao, ${ }^{\circ}$ Analytical formulae for the coupling coef®cient $\beta$ between a waveguide and a travelling wave structure ${ }^{\circ}, \mathrm{Nucl}$. Instr. and Meth., A330 (1993) p. 306.

[5] N. Holtkamp, ${ }^{\circ} \mathrm{S}$-band test accelerator ${ }^{\circ}$, Proceedings of the LC93, Oct. 13-21 (1993) Stanford, SLAC-436, 1994.

[6] N. Kroll, K. Thompson, K. Bane, R. Gluckstern, K. Ko, R. Miller, and R. Ruth, ${ }^{\circ}$ Higher order mode damping for a detuned structure ${ }^{\circ}$, SLAC-PUB-6624, Aug. 1994. 